\title{
Single-institution analysis of willingness of waitlist patients to undergo kidney transplantation with expanded criteria brain-dead donors
}

\author{
Jung Ja Hong ${ }^{1}$, Sae Rom Lee ${ }^{1}$, Ah Young Lee ${ }^{1}$, Ji Won Woo ${ }^{1}$, Seon Bin Park ${ }^{1}$, Shin Hwang² , Young Hoon Kim ${ }^{3}$ \\ ${ }^{1}$ Organ Transplantation Center, Asan Medical Center, University of Ulsan College of Medicine, Seoul, Korea \\ ${ }^{2}$ Department of Liver Transplantation and Hepatobiliary Surgery, Asan Medical Center, University of Ulsan College of Medicine, Seoul, Korea \\ ${ }^{3}$ Department of Kidney and Pancreas Transplantation Surgery, Asan Medical Center, University of Ulsan College of Medicine, Seoul, Korea
}

Background: The waitlist period for kidney transplantation (KT) has been increasing due to intractable shortage of brain-dead donors in Korea. To solve this organ shortage issue, KT cases with expanded criteria brain-dead donor (ECD) organs have been increasing. This study intended to analyze the willingness of waitlist patients to undergo KT with ECD in a single institution in order to use as the basic data for selecting and managing patients waiting for deceased donor KT (DDKT).

Methods: We carried out a survey with 1,069 patients who were enrolled at the waitlist for DDKT from 2015 to 2019 at a single institution. They were asked whether they were willing to undergo KT with ECD of type 1 and 2 . The answers were analyzed through descriptive statistics. ECD type 1: age over 60 years, but serum creatinine is normal ( $<1.5 \mathrm{mg} / \mathrm{dL}$ ) without hypertension or diabetes and type 2: serum creatinine is higher than $3.0 \mathrm{mg} / \mathrm{dL}$ due to acute kidney injury, under continuous renal replacement therapy to improve acute renal failure, or serum creatinine is normal $(<1.5 \mathrm{mg} / \mathrm{dL})$ but over 60 years of age with hypertension or diabetes. Results: Of them, 390 patients (36.0\%) refused ECD type 1. In contrast, other 345 patients (32.0\%) answered to undergo KT with deceased donors over 60 years, but they did not want KT with donors over 70 years; 231 (22.0\%) patients did not have a limit in the donor age; and 103(10\%) patients hesitated or avoided answers. As a result, $576(52.6 \%)$ of 1,096 patients were willing to undergo KT with ECD type 1. The proportions of such affirmative-answer group according to the patient age were $196(34.1 \%)$ in their 50s, $171(29.7 \%)$ in their 60s, $115(19.9 \%)$ in their 40s, 57 (9.9\%) in their 30s, 21 (3.7\%) in their 70s, $14(2.4 \%)$ in their 20s and three $(0.30 \%)$ in their teens. Meanwhile, 538 patients (50.3\%) refused to undergo KT with ECD type 2. Other 419 patients (39.2\%) were willing to undergo KT with ECD type 2; and 112 patients (10.5\%) avoided answer or did not responded. In the 419 patients who expressed their willingness to undergo KT with ECD type 2, the waitlist periods were $\geq 1$ year in 135 patients, $\geq 2$ years in 110 , $\geq 3$ years in $83, \geq 4$ years in 58 , and $\geq 5$ years in 33 . Their distributions by age were 148 patients over 50 s, 116 patients over 60 s and 70s, 93 patients over 40s, 52 patients over 30s, and 10 patients over 20s. Among the 576 patients who expressed their willingness to undergo KT with ECD type 1, 322 (55.9\%) answered to undergo any type of ECD DDKT.

Conclusions: We believe that the results of this study are helpful to select and manage the DDKT candidates quickly and efficiently when occurrence of ECD is notified. It is necessary to establish practical protocols and to improve awareness toward KT with ECD.

Corresponding author: Jung Ja Hong

E-mail: hj25009@hanmail.net

\section{(C) The Korean Society for Transplantation}

This is an Open Access article distributed under the terms of the Creative Commons Attribution Non-Commercial License (http://creativecommons.org/licenses/by-nc/4.0/) which permits unrestricted non-commercial use, distribution, and reproduction in any medium, provided the original work is properly cited. 